\title{
Image Denoising Using Multiwavelet Transform with Different Filters and Rules
}

\author{
https://doi.org/10.3991/ijim.v15i15.24183 \\ Muna Majeed Laftah ${ }^{\bowtie)}$ \\ University of Baghdad, Baghdad, Iraq \\ muna.majeed@coeduw. uobaghdad.edu.iq
}

\begin{abstract}
Image denoising is a technique for removing unwanted signals called the noise, which coupling with the original signal when transmitting them; to remove the noise from the original signal, many denoising methods are used. In this paper, the Multiwavelet Transform (MWT) is used to denoise the corrupted image by Choosing the HH coefficient for processing based on two different filters Tri-State Median filter and Switching Median filter. With each filter, various rules are used, such as Normal Shrink, Sure Shrink, Visu Shrink, and Bivariate Shrink. The proposed algorithm is applied Salt\&pepper noise with different levels for grayscale test images. The quality of the denoised image is evaluated by using Peak Signal to Noise Ratio (PSNR). Depend on the value of PSNR that explained in the result section; we conclude that the (Tri-State Median filter) is better than (Switching Median filter) in denoising image quality, according to the results of applying rules the result of the Shrinking rule for each filter shows that the best result using first the Bivariate Shrink.
\end{abstract}

Keywords - denoising, multiwavelet, thresholding, soft thresholding

\section{Introduction}

Image denoising is to remove noise from a noisy image to restore the true image. It is essential to retrieve a correct image with no defects generated during capturing a real-world scene or throw the instrument used to get the data to establish the noise $[1,2]$. The noise can be generated through electronic transmission during transmitting the image. The noise was unwanted fluctuating of information that decreases the visually and quality of the image [3]. The image is represented as $2 \mathrm{D}$ function $\mathrm{f}(\mathrm{x}, \mathrm{y})$, such that $\mathrm{x}, \mathrm{y}$ are two coordinates of the matrix. The values of these coordinates are called the intensity of the image. Images consist of several elements; these elements are called picture elements (pixels), the noises will be fluctuating the pixels. Many kinds of noises exist in the image, such as Salt\&pepper, Gaussian noise, Shot noise, Speckle noise, and white noise [4]. There are many techniques for noise reduction and removal from the image that are depending and using individual filtering. Noise removal and reduction must be done without losing much detail from the processed image; this goal is achieved using a mathematical function called transform to spread the image into different frequency (Sub-bands) and effectively reduce the noise. 


\section{Related work}

In [5], The authors proposed a hybrid approach to remove the noise from the image. By decomposing into four LL, LH, HL, and HH levels and applying the conventional denoising methods for the high-frequency information such as Wiener filter, Median filter, and wavelet soft threshold. Wavelet-based decomposition before denoising. The quality of the image is enhanced in terms of detail and edge and removing the noise. The evaluation of the proposed method is measured in terms of PSNR, MSE, and structural similarity. The proposed algorithm concludes that the method has a better denoising effect on medical images.

In this paper [6], the denoising method is based on the Rotated wavelet filter to increase the performance of the denoising algorithm by considering energy analysis if the energy test of an image is passed, which will support performance. The images used to test the proposed algorithm are general and medical X-Ray images. The results for the proposed algorithm according to PSNR show a good computational complexity which is similar to the $2 \mathrm{D}$ discrete wavelet transform domain platform and less execution time.

In [3], the author proposed image denoising based on Daubechies basis functions. They used two proposed methods using a soft and hard threshold to change the wavelet transform coefficient values. The quality of image reconstruction depends on the noise intensity and image size, provided minimal error. A soft and hard threshold was shown that soft threshold improves the performance of denoising for the DWT compared with hard thresholding. All coefficients for a noisy image are corrected to preserve the regularity of the signal after reconstruction; the soft threshold is the best method for wavelet denoising. From the results, the best result for image denoising is achieved during the wavelet db8 with minimum error. The Root-Mean-Square error (RMS) is used to compute the error.

In [1], introduced denoise the image components and kept the geometry of image instead of working directly in the image, they proposed a denoising method, by combining the parts into a single vector-valued function to which apply a denoising method (vector-valued) or treat them separately applying the same denoising method. Still, with different parameters, they produced working on framework best than image directly according to denoising process both in terms of Peak signal-to-noise ratio and structural similarity index metrics.

\section{Multiwavelet transform}

Multiwavelet is a wavelet with different scaling functions; it is better than wavelet in the domain of image denoising, compression, and classification because there are many characteristics like symmetry, orthogonally and short support these properties needed to increase the efficiency of 2D-image, multi-wavelet contains multiple scaling instead of single scaling function using wavelet [7].

The main approach for the multi-wavelet is multi_resolution decomposition; sixteen sub-bands are produced after the first level of decomposition, while in wavelet, four sub-bands. The standard Multi-Resolution Analysis (MRA) for scalar wavelet uses a single scaling function denoted as $\varphi(t)$ and single wavelet $\psi(t),[8-10]$.

$$
\Psi(t)=\sqrt{2} \Sigma_{k} H_{k} \Phi(2 t-k)
$$


The coefficients of the $1^{\text {st }}$ level of multi-wavelet $\mathrm{W}_{1, \mathrm{k}}$ can be get by high-pass filtering and down-sampling:

$$
\mathrm{W}_{1, \mathrm{k}}=\Sigma_{\mathrm{m}} \mathrm{H}_{\mathrm{m}-2 \mathrm{k}} \mathrm{V}_{0, \mathrm{~m}}
$$

Multi-wavelet decomposition can be found to indicate the recombination of the scaling factor:

$$
\begin{aligned}
\mathrm{V}_{\mathrm{j}, \mathrm{k}} & =\Sigma_{\mathrm{m}} \mathrm{G}_{\mathrm{m}-2 \mathrm{k}} \mathrm{V}_{\mathrm{j}-1, \mathrm{~m}} \\
\mathrm{~W}_{\mathrm{j}, \mathrm{k}} & =\Sigma_{\mathrm{m}} \mathrm{H}_{\mathrm{m}-2 \mathrm{k}} \mathrm{V}_{\mathrm{j}-1, \mathrm{~m}}
\end{aligned}
$$

Figure 1 shows a one-level multi-wavelet decomposition [7].

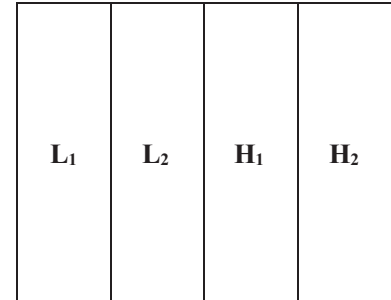

(a)

\begin{tabular}{|l|l|l|l|}
\hline $\mathbf{L}_{1} \mathbf{L}_{1}$ & $\mathrm{~L}_{1} \mathrm{~L}_{2}$ & $\mathrm{~L}_{1} \mathrm{H}_{1}$ & $\mathrm{~L}_{1} \mathrm{H}_{2}$ \\
\hline $\mathrm{L}_{2} \mathrm{~L}_{1}$ & $\mathrm{~L}_{2} \mathrm{~L}_{2}$ & $\mathrm{~L}_{2} \mathrm{H}_{1}$ & $\mathrm{~L}_{2} \mathrm{H}_{2}$ \\
\hline $\mathrm{H}_{1} \mathrm{~L}_{1}$ & $\mathrm{H}_{1} \mathrm{~L}_{2}$ & $\mathrm{H}_{1} \mathrm{H}_{1}$ & $\mathrm{H}_{1} \mathrm{H}_{2}$ \\
\hline $\mathrm{H}_{2} \mathrm{~L}_{1}$ & $\mathrm{H}_{2} \mathrm{~L}_{2}$ & $\mathrm{H}_{2} \mathrm{H}_{1}$ & $\mathrm{H}_{2} \mathrm{H}_{2}$ \\
\hline
\end{tabular}

(b)

Fig. 1. First Level decomposition of Multiwavelet image sub-bands

(a). Horizontal filtering direction (b). Vertical filtering direction after horizontal direction

Two low pass sub-bands and two high pass sub-bands for each dimension are obtained from the decomposition of the multi-wavelet. Figure 1 illustrated the construction of the sub-bands after the first level of multi-wavelet decomposition, where sixteen sub-bands are resulted [8].

\section{Thresholding techniques}

It is a non-linear method based on one transform coefficient at a time; after determining the threshold, each coefficient value will set to zero if it is less than the threshold value; otherwise, it will be updated or kept with no change. The coefficients of the large value represent the signal information, while the small coefficient values are dominated by noise. The reconstruction process is done by changing small coefficients based on a threshold value (noise coefficients) with zero then applying the inverse of the transform to reconstruct the image with less noise [11].

\subsection{Soft thresholding}

A soft thresholding can be defined as following:

$$
T_{£}^{\text {Soft }}(x[i, j])=\left\{\begin{array}{c}
\operatorname{sgn}(x[i, j])(\mid x[i, j \mid-£]),|x[i, j]|>£ \\
0|x[i, j]| \leq £
\end{array}\right.
$$


Where $T$ is the value of the threshold.

Figure 2 illustrates the soft threshold.
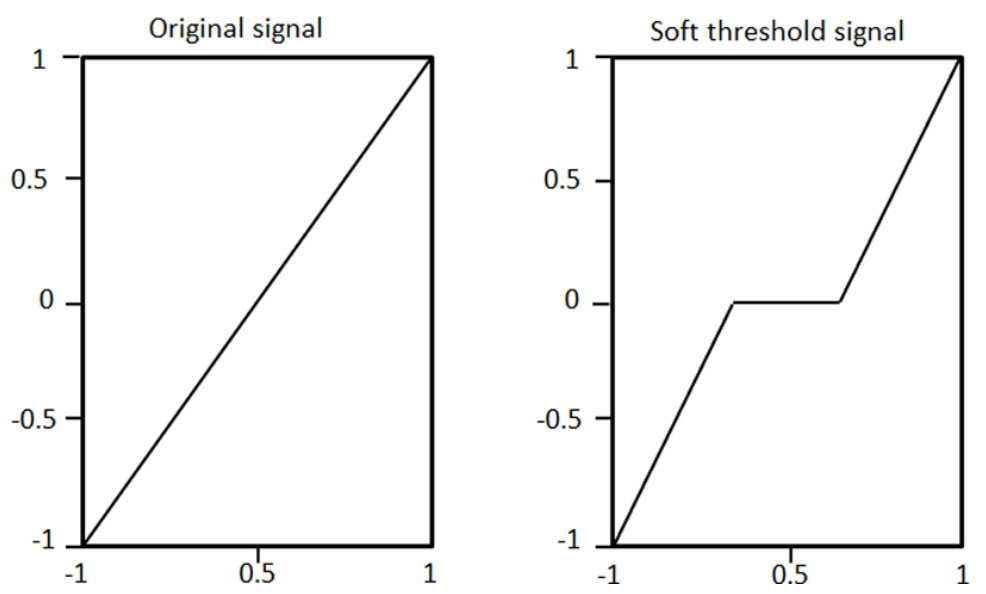

Fig. 2. Soft threshold with $£=0.4$

\section{$5 \quad$ Filters for noise removal}

\subsection{Tri-state median filter}

When a combination was performed between the Center Weighted Median (CWM) filter and Standard Median Filter (SM) to indicate if a pixel is destroyed previously, to apply unconditionally filter as demonstrated in Figure 3, the result of the Tristate Median Filter (TRIMF) is obtained correspond to one of three possible states, the first case (i.e., the pixel is noise-free) for the value of the original pixel, the second case is the Standard Median Filter (SM) (i.e., the corrupted pixel), and the last CWM filtered output (i.e., the uncorrupted pixel) as illustrated in Figure $3[11,12]$.

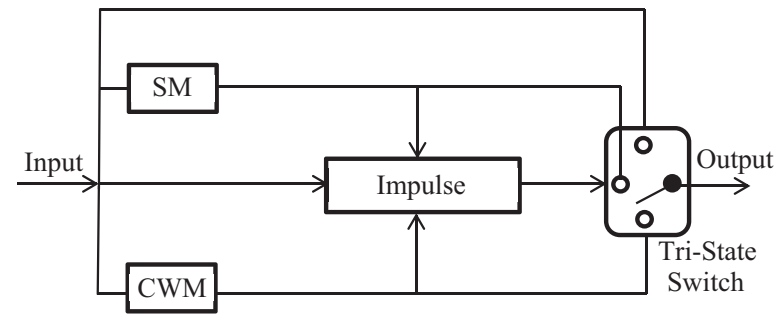

Fig. 3. Tristate median filter 


\subsection{Switching median filter}

These days, Switching Median Filter (SMF) is the most popular median filtering scheme; it is also known as (decision-based median filter). SMF attempts to reduce the unwanted change of undamaged pixels with the filter, so for beating this difficult point, SMF sees the pixel for input has been modified or not by the noise by changing the intensity of the noisy pixel. In contrast, it leaves the other pixels which have no modification. Usually, the SMF is used for two steps: detecting noise and the other step for deleting the noise [12].

The result from the first step of SMF is making a mask for noise $\mathrm{M}$ which is a binary mask and can be explained as:

$$
M(i, j)=\left\{\begin{array}{lr}
1: & \text { impulsenoise candidate } \\
0: & \text { otherwise }
\end{array}\right.
$$

\section{Thresholding selection}

\subsection{Normal Shrink}

The threshold value for the Normal shrink (NS) representing the optimal value is given by [13].

$$
T N=\frac{\lambda \sigma v^{2}}{\sigma v}
$$

where $\lambda$ is calculated by equation (8).

$$
\lambda=\sqrt{\log \left(\frac{L_{k}}{j}\right)}
$$

Where $L_{k}$ represents the sub-band length at the Kth scale, $(j)$ represents the complete number for decomposition. $(\sigma)$ represents the estimation for Noise Variance. (NS) also computes soft threshold based on the threshold TN, which is performed using equation (7).

\subsection{Sure Shrink}

Donoho and Johnstone proposed Stein's Unbiased Risk Estimator (SURE) as an approach for a threshold Chooser; this method is named Sure Shrink, which combines two thresholds, the universal and the SURE thresholds. It assigns a (tj) value for every resolution level (j) in the (wavelet transform). The objective of Sure Shrink reducing the Mean Squared Error (MSE), which is defined as the following [14].

$$
M S E=\frac{1}{n^{2}} \sum_{i}^{n} j=(z(i, j)-s(i, j))^{2}
$$

Where $z(i, j)$ is the signal free from noise and $(n)$ represents the size of the signal, by thresholding, the empirical Multiwavelet coefficients Sure Shrink will destroy the noise. The threshold $t^{*}$ of Sure Shrink is defined as: 


$$
t^{*}=\min (t, \sigma \sqrt{2 \log n})
$$

Such that ( $\mathrm{t}$ ) represented the value that reduces 'Stein's Unbiased Risk Estimator', $\sigma$ is the noise variance, and (n) represents the size of the image.

\subsection{Visu Shrink}

Visu Shrink is also called a universal threshold; it is introduced by 'Donoho and Johnstone'. VisuShrink is performed by applying the global threshold. This threshold is computed by

$$
t=\sigma \sqrt{2 \log n}
$$

Such that $(\sigma)$ is the variance of the noise, (n) is the total number of image pixels [15]. The assessment for the noisy level $\sigma$ is determined to depend on the computed median value for the absolute deviation presented by equation (12)

$$
\sigma=\frac{\operatorname{median}\left(\left\{a b s\left(g_{i-1}, k\right): k=0,1, \ldots \ldots \ldots 2^{j-1}\right\}\right)}{0.6745}
$$

Where $g_{j-1}$ and $(k)$ refer to the more coefficients details in the multi-wavelet transform.

The asymptotically produces a Mean Square Error (MSE) assessment as (m) tends to infinity. If (n) increased, we get a bigger and bigger threshold, smoothen the image.

\subsection{Bivariate Shrink}

The Bivariate Shrink is a new shrinking function that depends on two factors, the parent and the coefficient, for producing a good result of multi-wavelet image denoising. It is based on the Bayesian estimation to compute the dependency between the parent and coefficient $[16,17]$. For the following equation, $z_{2}$ refers to the parent of $z_{1}\left(z_{2}\right.$ is the coefficient of multi-wavelet at the same position as $\left.z_{1}\right)$, then

$$
\begin{aligned}
& x_{1}=z_{1}=n_{1} \\
& x_{2}=z_{2}+n_{2}
\end{aligned}
$$

Where $\mathrm{n}_{1}$ and $\mathrm{n}_{2}$ are noise samples, $\mathrm{x}_{1}$ and $\mathrm{x}_{2}$ are the noise of $\mathrm{z}_{1}$ and $\mathrm{z}_{2}$, as:

$$
\begin{gathered}
x=z+n \\
x=\left(z_{1}, z_{2}\right) \\
z=\left(z_{1}, z_{2}\right) \\
n=\left(n_{1}, n_{2}\right)
\end{gathered}
$$

The coefficient estimation could be evaluated based on the noise densities and prior density of the multi-wavelet coefficient. Suppose the noise is 'Gaussian' so it can be written as:

$$
\operatorname{Pn}(n)=\frac{1}{2} \pi\left(\sigma n^{2}\right) e^{\left(-n_{1}^{2}+n_{2}^{2} / 2 \sigma n^{2}\right)}
$$


The above equation (13) is equal to resolve the following equations

$$
\begin{aligned}
& y_{1}-\frac{w_{1}}{\sigma n^{2}}+f_{1}(w)=0 \\
& y_{2}-\frac{w_{2}}{\sigma n^{2}}+f_{2}(w)=0
\end{aligned}
$$

$f_{1}$ and $f_{2}$ refer to the derivative of $f(w)$ corresponding to $w_{1}$ and $w_{2}$ consequently.

\section{Proposed denoising algorithm}

In this paper, a proposed method is designed to remove the noise from the digital image; firstly, a preprocessing step is performed (TSMF \& SMF) to detect and enhanced the corrupted pixel, the ratio of denoising based on the noise level, for the output filtering image a multi-wavelet is performed for removing the rest noise, high frequency contains both the details and the noise while low frequency contains the energy so that soft thresholds are applied for high frequency, the following block diagram shows the main steps of the proposed algorithm.

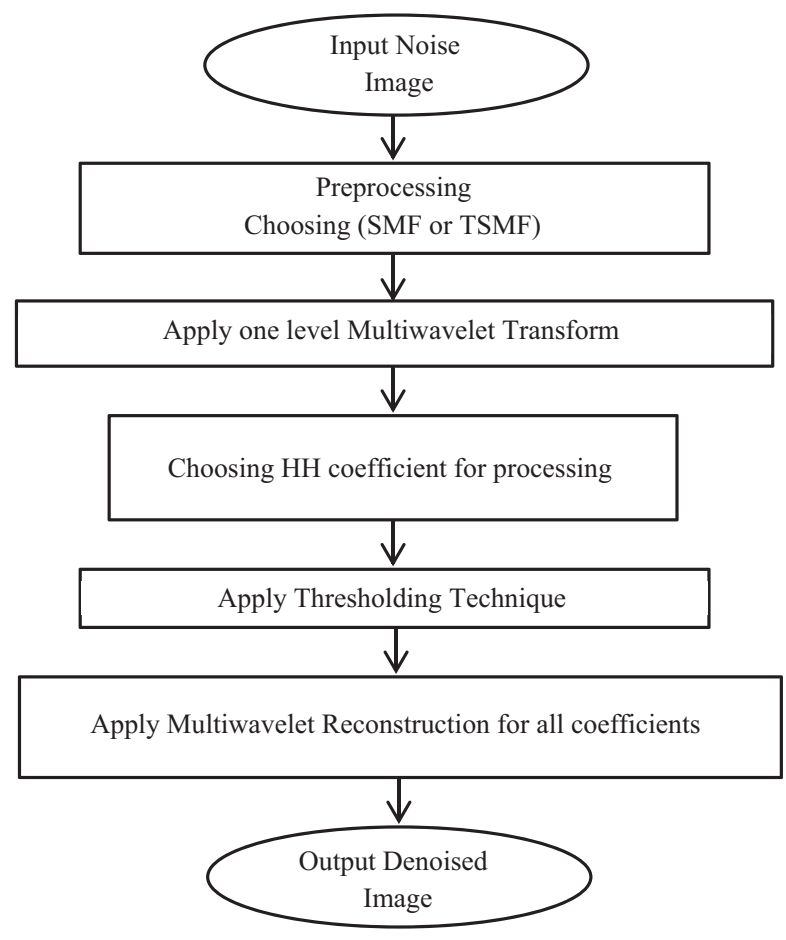

Fig. 4. Block diagram for the proposed system 


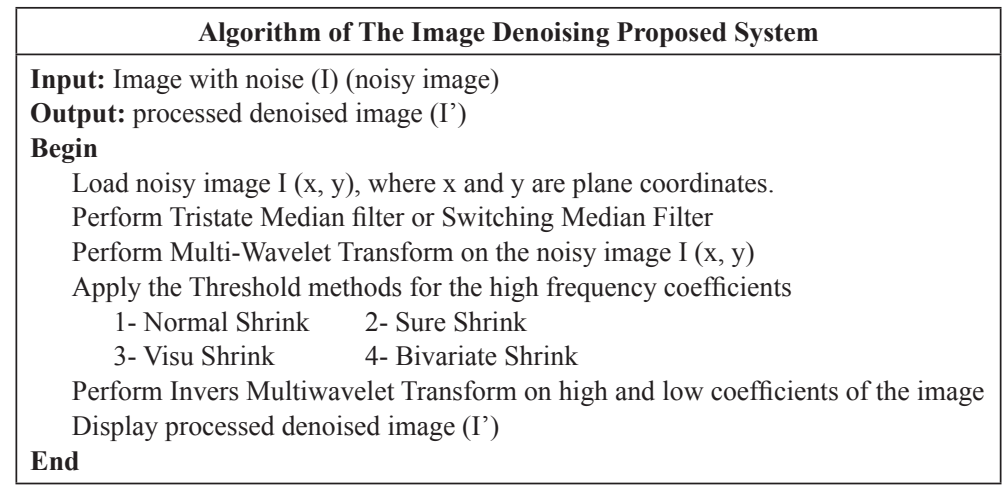

\section{Implementation and results}

The quality of the denoised image is evaluated by using Peak Signal to Noise Ratio (PSNR) as in [18-20]. The mathematical representation of the PSNR is as follows:

$$
\begin{gathered}
M S E=\left(1 /\left(m^{*} n\right)\right) * \operatorname{sum}\left(\operatorname{sum}\left((f-g)^{2}\right)\right) \\
P S N R=20 * \log (\max (\max (f))) /\left((M S E)^{0.5}\right)
\end{gathered}
$$

Where $\mathrm{f}$ represents the matrix data of our original image, $g$ represents the matrix data of the denoised image, $m$ represents the numbers of rows of pixels of the images, $n$ represents the number of columns of pixels of the image, and $\max (f)$ is the maximum signal value that exists in our original image.

The implementation and results are illustrated in Tables 1 and 2. Three different images (Cat, Actors, and Fruits) of size 512*512 are used as test images for two different filters (Tri-state Median filter and SMF). The noise added to the test image is (Salt\&pepper) as shown in Figure 5. Different amounts of noise factor $=5 \%, 10 \%, 20 \%$, and $30 \%$ are added to the test image using different filters with different Shrinking Methods Normal Shrink, Sure Shrink, Visu Shrink, and Bivariate Shrink.

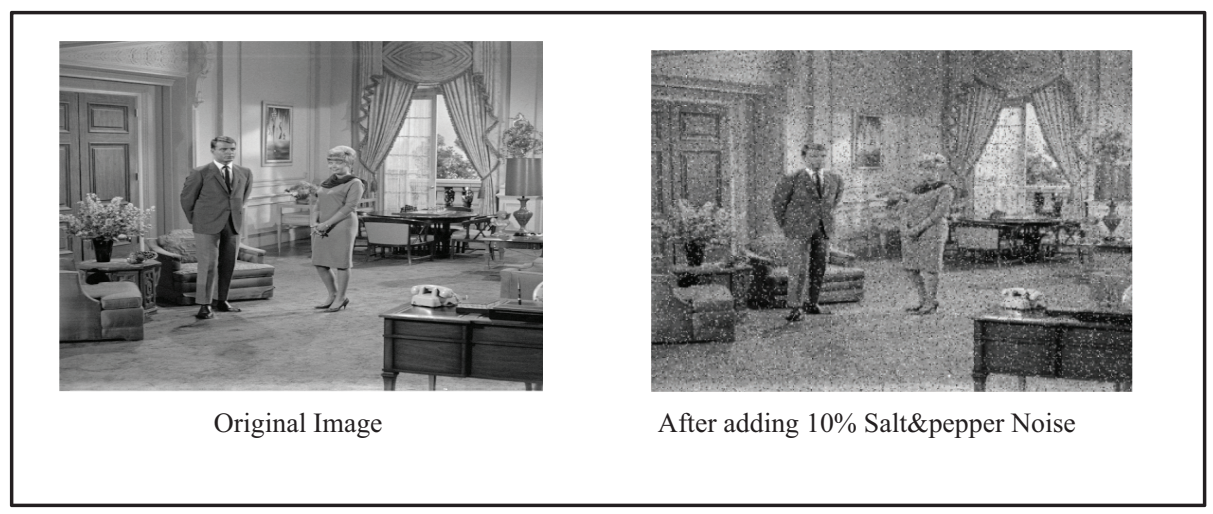

Fig. 5. Adding Salt\&pepper noise 
Table 1. The results test for the Tri-State filter

\begin{tabular}{|c|c|c|c|c|c|}
\hline \multirow[b]{2}{*}{ Image name } & \multirow[b]{2}{*}{$\begin{array}{c}\text { Noise } \\
\text { factor(f) }\end{array}$} & \multicolumn{4}{|c|}{ PSNR using Tri-State } \\
\hline & & $\begin{array}{c}\text { Bivariate } \\
\text { Shrink }\end{array}$ & $\begin{array}{c}\text { Normal } \\
\text { Shrink }\end{array}$ & $\begin{array}{c}\text { Sure } \\
\text { Shrink }\end{array}$ & $\begin{array}{c}\text { Visu } \\
\text { Shrink }\end{array}$ \\
\hline & 5 & 39.35 & 38.72 & 38.31 & 28.81 \\
\hline & 10 & 38.12 & 37.37 & 37.04 & 27.34 \\
\hline & 20 & 34.72 & 34.41 & 34.68 & 25.06 \\
\hline & \multicolumn{4}{|c|}{ Cat } & 23.71 \\
\hline \multirow{4}{*}{ 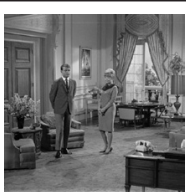 } & 5 & 37.21 & 36.81 & 36.59 & 25.94 \\
\hline & 10 & 35.73 & 35.24 & 34.97 & 24.88 \\
\hline & 20 & 33.08 & 32.39 & 32.41 & 22.75 \\
\hline & 30 & 30.51 & 30.47 & 30.26 & 20.69 \\
\hline \multicolumn{6}{|l|}{ Actors } \\
\hline \multirow{4}{*}{ 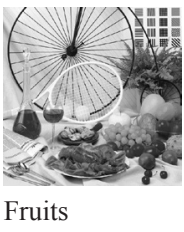 } & 5 & 37.82 & 37.86 & 37.17 & 27.68 \\
\hline & 10 & 36.84 & 36.23 & 35.70 & 26.09 \\
\hline & 20 & 33.93 & 33.58 & 32.01 & 23.12 \\
\hline & 30 & 31.27 & 31.02 & 29.58 & 20.34 \\
\hline
\end{tabular}

Table 2. The results test for the SMF filter

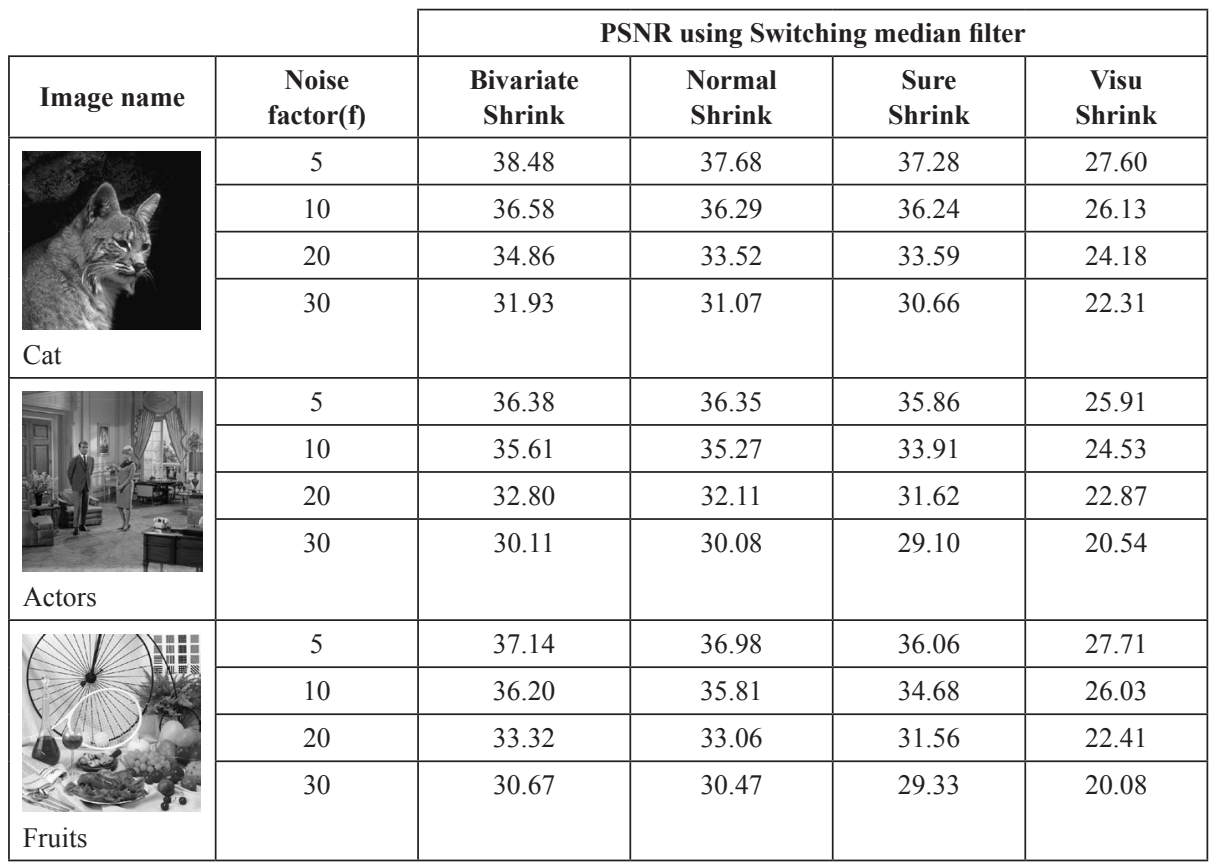


The PSNR values plots for three test images using TSMF and SMF are illustrated in Table 3 with the Bivariate Shrink, Normal Shrink, Sure Shrink, and Visu Shrink methods.

Table 3. The plot for TSMF and SMF where the $x$-axis represents the method type and $y$-axis the PSNR

\begin{tabular}{|c|c|}
\hline \multicolumn{2}{|c|}{ PSNR using TSMF } \\
\hline \multicolumn{1}{|c|}{ Cat image } \\
\hline \\
\hline
\end{tabular}
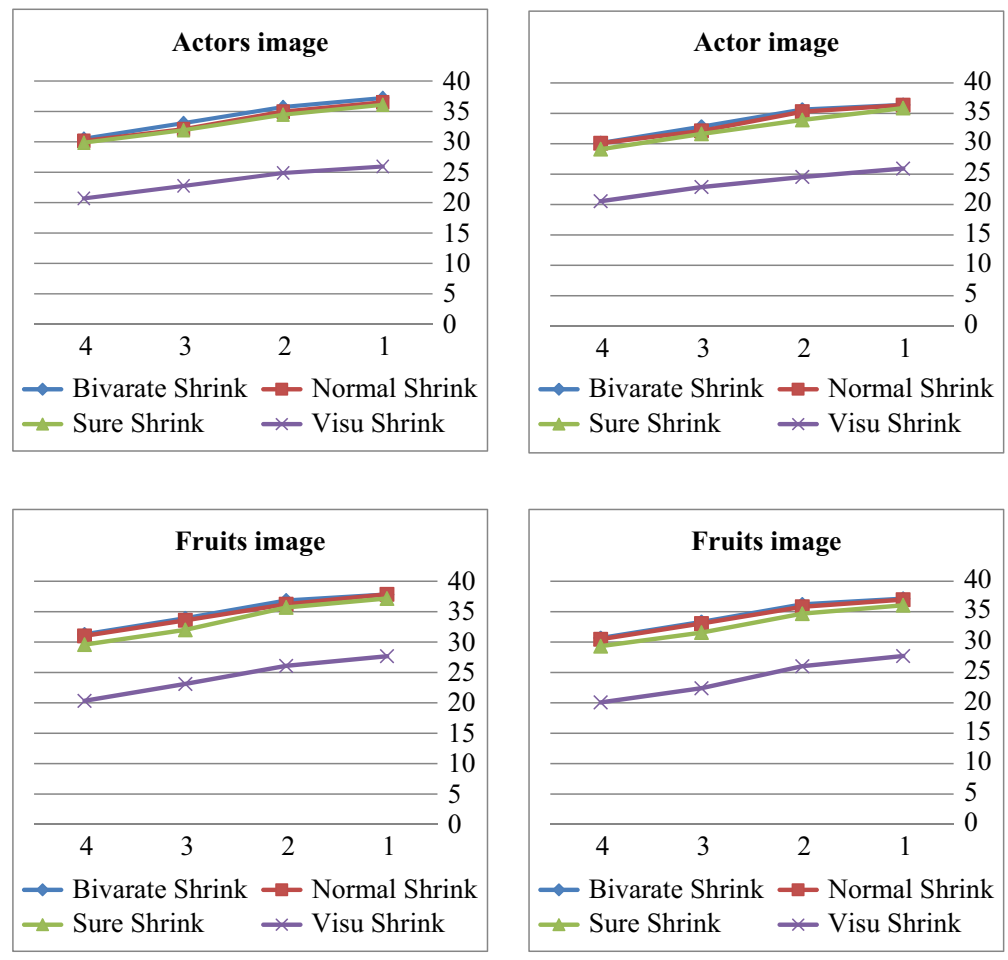


\section{Conclusion}

This paper presents the results of denoising images using different filters, with each of them used different Shrinking rules. We conclude from these result that the (TSMF) is better than (SMF) in denoising image quality; at the same time, the result of the Shrinking rule for each filter shows that the best result using first the Bivariate Shrink, the second was Normal Shrink, the third was Sure Shrink and the last was Visu Shrink.

\section{References}

[1] G. Ghimpeteanu, T. Batard, M. Bertalmio, and S. Levine, "A decomposition framework for image denoising algorithms," IEEE Trans. on Image Process., vol. 25, no. 1, pp. 388-399, 2016. https://doi.org/10.1109/TIP.2015.2498413

[2] L. Fan, F. Zhang, H. Fan, and C. Zhang, "Brief review of image denoising techniques," Visual Computing for Industry, Biomedicine, and Art," vol. 7, no. 2, 2019. https://doi.org/ 10.1186/s42492-019-0016-7

[3] S. Alauldeen, S. Rafid, and H. Jabbar, "Wavelet-Based Denoising Of Images," Engineering and Technology Journal, vol. 37, no. 02, 2019.

[4] G. Kaur and R. Kaur, "Image Denoising using Wavelet Transform and Various Filters,” Int. J. Res. Comput. Sci., vol. 2, no. 2, pp. 15-21, 2012. https://doi.org/10.7815/ijorcs.22.2012.017

[5] L. M. Satapathy, P. Das, A. Shatapathy, and A. K. Patel, "Bio-Medical Image Denoising using Wavelet Transform," Int. J. of Rece. Tech. and Engin.(IJRTE), vol. 8, no. 1, 2019.

[6] K. V. Thakur, P. G. Ambhore, and A. M. Sapkal, "Novel Technique for Performance Improvement of the Wavelet based Denoising Algorithms using Rotated Wavelet Filters," Procedia Comput. Sci., vol. 79, pp. 499-508, 2016. https://doi.org/10.1016/j.procs.2016.03.064

[7] S. Vaid, P. Singh, and C. Kaur, "Classification of Human Emotions using Multiwavelet Transform based Features and Random Forest Technique," Indian J. Sci. Technol., vol. 8, no. 28, 2015. https://doi.org/10.17485/ijst/2015/v8i1/70797

[8] M.Iman, "Image Steganography by Using Multiwavelet Transform," Baghdad Sci. J., vol. 11, no. 2, pp. 275-283, 2014. https://doi.org/10.21123/bsj.11.2.275-283

[9] Q. Hamarsheh, O. Daoud, and S. Saraireh, "Wavelet entropy algorithm to allocate the extreme power peaks in WiMax systems," Int. J. Interact. Mob. Technol., vol. 8, no. 4, pp. 14-19, 2014. https://doi.org/10.3991/ijim.v8i4.3766

[10] Z. G. Wang, W. Wang, and B. Su, "Multi-sensor image fusion algorithm based on multiresolution analysis,” Int. J. Online Eng., vol. 14, no. 6, pp. 44-57, 2018. https://doi.org/10.3991/ ijoe.v14i06.8697

[11] A. Al Jumah, "Denoising of an Image Using Discrete Stationary Wavelet Transform and Various Thresholding Techniques,” J. Signal Inf. Process., vol. 04, no. 01, pp. 33-41, 2013. https://doi.org/10.4236/jsip.2013.41004

[12] E. J. Leavline and D. A. A. G. Singh, "Salt and Pepper Noise Detection and Removal in Gray Scale Images: An Experimental Analysis," Int. J. Signal Process. Image Process. Pattern Recognit., vol. 6, no. 5, pp. 343-352, 2013. https://doi.org/10.14257/ijsip.2013.6.5.30

[13] A. Khmag, A. Rahman, S. Abdul, and R. Al-haddad, "A Detailed Study on Image Denoising Algorithms by Using the Discrete Wavelet Transformation," Int. J. of comput. Sci. and Technol. (IJCST), vol. 8491, pp. 17-24, 2014.

[14] H. Om and M. Biswas, "An Improved Image Denoising Method Based on Wavelet Thresholding,” J. Signal Inf. Process., vol. 03, no. 01, pp. 109-116, 2012. https://doi.org/ $\underline{10.4236 / \text { jsip.2012.31014 }}$ 
[15] M. Neelima and M. Pasha, "Wavelet Transform Based On Image Denoising Using Thresholding Techniques," Int. J. Adv. Res. Comput. Commun. Eng., vol. 3, no. 9, pp. 2278-1021, 2014, [Online]. Available: www.ijarcce.com.

[16] N. Dewangan and A. Das Goswami, "Image denoising using wavelet thresholding methods," Int. J. of Engg. Sci. \& Mgmt. (IJESM), vol. 2, no. 2, pp. 271-275, 2012.

[17] H. Najm, H. K. Hoomod, and R. Hassan, "A New WoT Cryptography Algorithm Based on GOST and Novel 5d Chaotic System," Int. J. Interact. Mob. Technol., vol. 15, no. 2, pp. 184-199, 2021. https://doi.org/10.3991/ijim.v15i02.19961

[18] H. A. Naman, N. A. Hussien, M. L. Al-dabag, and H. T. S. AlRikabi, "Encryption System for Hiding Information Based on Internet of Things," Int. J. Interact. Mob. Technol., vol. 15, no. 2, pp. 172-183, 2021. https://doi.org/10.3991/ijim.v15i02.19869

[19] P. Zhao, X. Zhao, and C. Zhao, "Image denoising based on bivariate distribution," Symmetry in Artificial Visual Perception and Its Application, vol. 12, no. 11, pp. 1-10, 2020. https://doi.org/10.3390/sym12111909

[20] I. A. Aljazaery, H. T. S. Alrikabi, and M. R. Aziz, "Combination of hiding and encryption for data security,” Int. J. Interact. Mob. Technol., vol. 14, no. 9, pp. 34-47, 2020. https://doi.org/ $\underline{10.3991 / i j i m . v 14 i 09.14173}$

\section{Author}

Muna Majeed Laftah is presently Asst. Prof in Baghdad University/College education for women/Computer Science department. She received his B.Sc. degree in computer science in 1995 from the Al Technology University in Baghdad, Iraq. Her M.Sc. degree in computer science focuses on multimedia security from the Iraqi Commission for Computers and Informatics/Iraq in 2003. Her Ph.D. degree in computer science from Al technology University in Baghdad, Iraq/2017. Her current research interests include 3D security, encryption of multimedia. E.mail: muna.majeed@,coeduw. uobaghdad.edu.iq

Article submitted 2021-05-27. Resubmitted 2021-07-02. Final acceptance 2021-07-04. Final version published as submitted by the authors. 